\title{
RELATIONSHIP MODEL BETWEEN NIGHTLIGHT DATA AND FLOOR AREA RATIO FROM HIGH RESOLUTION IMAGES
}

\author{
Meichun $\mathrm{Yan}^{\mathrm{a}}{ }^{*}$, Lingli $\mathrm{Xu}{ }^{\mathrm{a}}$ \\ a College of Earth Science and Engineering, Hohai University, Nanjing, China; \\ *Corresponding author, e-mail: yanmeichun@hhu.edu.cn
}

Commission VI, WG VI/4

KEY WORDS: Relationship model, Nightlight VIIRS/NPP, ZY-3 images, Floor area ratio, Administrative street level

\begin{abstract}
:
It is a hotpot that extraction the floor area ratio from high resolution remote sensing images. It is a development trend of using nightlight data to survey the urban social and economic information. This document aims to provide a conference relationship model for VIIRS/NPP nightlight data and floor Area Ratio from High Resolution ZY-3 Images. It shows that there is a lineal relationship between the shadow and the floor area ratio, and the $\mathrm{R}^{2}$ is 0.98 . It shows that there is a quadratic polynomial relationship between the floor area ratio and the nightlight, and the $\mathrm{R}^{2 \text { is }} 0.611$. We can get a conclusion that, VIIRS/NPP nightlights data may show the floor area ratio in an extent at level of administrative street.
\end{abstract}

\section{INTRODUCTION}

It is very important for ecological environment surveying to grasp the population activity spatial distribution style, the traditional source of population is population census, and relational administrative organization divisions is the basic unit to collect and manage population information, there are two problems in practical application(DC,2000): (1) the spatial resolution is low, although the population data is based on community census collected, but usually taking the county level as an unit in the population bulletin. (2the time resolution is low, for the national census is a systematic project, the data updating cycle is long for years, and so, it is impossible to provide real-time population data usually.

High-resolution satellite remote sensing data is more and more easier to obtained, it is very conventional in buildings or resident areas presentation, which contributes to population relational information interpretation (Kellner,2017), building floor ratio is an important index of population distribution as well as obvious information in high resolution remote sensing images of urban area. Research in buildings floor ratio push the application of high resolution remote sensing images forward and elevate its prospects. We can probe urban deeply and widely increasingly.

On the other hand, Night light data adds to be more widely used in a variety of social science research such as population spatial distribution and economic development. The OLS (Operational Line-scan System), launched by the Defense Meteorological Satellite Program, can perfectively detect human activities by nighttime intensity, with unparalleled advantages such as widely monitoring and fast updating. Since the DMSP / OLS nighttime light data itself infers information about the population lighting, roads lighting, and other population-activity information(Townsend,2010; Small,2013), it can be used to reflect a wide range of population density variance. For example, researchers found that there is a clear linear correlation between the light area and the population, and the model fit to 0.85 or more (Forbes,2011).

Many experiences indicate that DMSP / OLS nighttime light data has great potential for realizing population spatial data grid. But now, the satellite system NPP (NPOESS Preparatory Project) VIIRS (Visible infrared Imaging Radiometer) visible infrared imaging radiometer night light data has not yet so many been used to do population density analysis (Vermote,2014; Hillger,2013 ), while its spatial resolution is 500 meters, which is twice of DMSP/OLS.

It is a development trend of using nightlight data to survey the urban social and economic information. VIIRS/NPP, for its higher resolution as well as fine radiation discrimination and wider width than DMSP/OLS, needs more research attention on its application in urban society and urban economy. The union of high resolution images and the nightlight data will improve our knowledge on urban activity in detail and needs more research attention. This article research wants to answer the two questions: one is: can we get the floor area ratio from $\mathrm{ZY}-3$ images? The other question, what is the relationship between nightlight data and the floor area ratio, or, can NPP VIIRS data imply the building floor area ratio of urban in administrative street level? We try some tests to get the conclusion.

\section{STUDY AREA AND DATA}

\subsection{Study Area}

Taking Nanjing city as research area, and the relative information of fifty streets belong to administrative level of main urban area as analysis and modelling data. Nanjing is the 
centre of east China, it is the leader and the benchmarking in culture and education, as well as in economic field, the population of Nanjing city suffered witling down during the Second World War because of Nanjing Massacre, and it also experienced rapid increasing during the period of Reform and Opening in last 40 years (Qin, 2013). It is enjoying comfortable and wealthy in good vegetation and excellent ecology environment in partial districts, while it is suffering crowded and chaotic in some other areas too. It is very practical for us to know about the population activity distribution situation as a whole, as well as to research and analysis the relationship between the floor ratio from high resolution ZY-3 images and the VIIRS Nightlight data.

\subsection{Data}

Surveying the information of total building area of 34 plot of residential districts by the field surveying is used for inversion modelling built from shadow to the total built area, ZY-3 images(China's first civil high-resolution threedimensional mapping satellite independently, successfully launched at 9:17 on September 9, 2015, spatial resolution of PAN band is 2.1 meters, and multi-spectral resolution is 6 meters) obtained in 20120219 of Nanjing to obtain urban buildings shadow, VIIRS/NPP data of 12 months in 2015 to calculate the nightlight index, all streets administrative level contour vector maps of Nanjing city to get the contour range of the streets for statistic, additional 55 floor ratio data of mini residential districts from planning institute of Nan-jing city are used for test. The sample villages dispatch as shown in figure 1 .

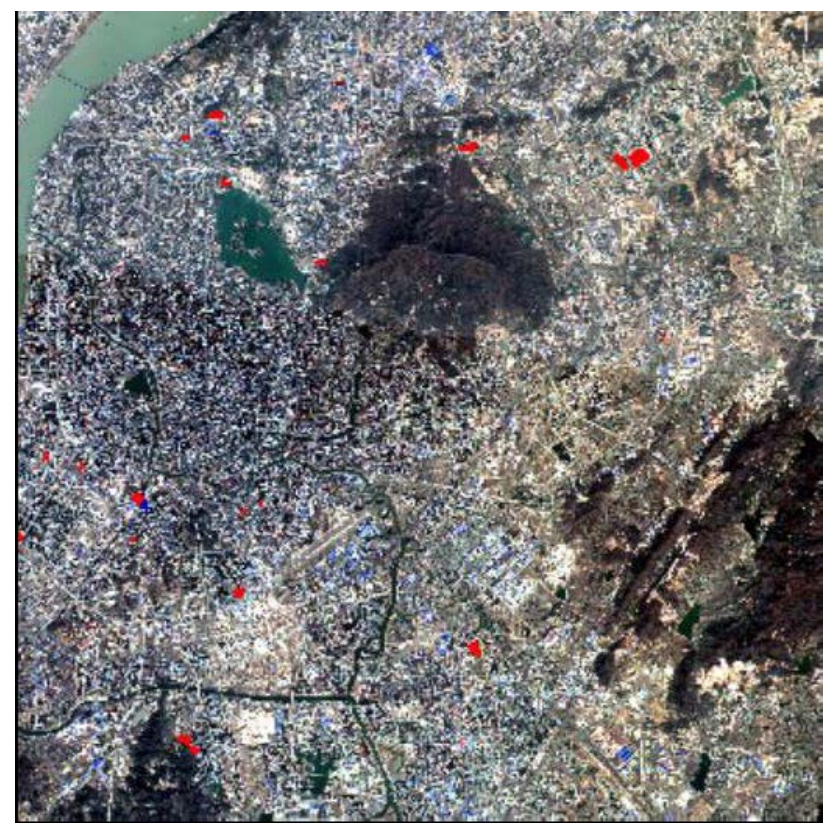

Figure 1. The sample villages dispatch (red area in the image)

\section{METHODOLOGY}

Two main steps are carried out in this research as following. Firstly, inversion of floor area ratio from high resolution ZY-3 images, secondly, the relationship modelling between floor area ratio inversed from ZY-3 images and the VIIRS nightlight data.

\subsection{Inversing of Floor Area Ratio}

We tried four absolutely necessary jobs to reach this target highly precisely, they are respectively: Gram-Schmidt images sharpening to improve the spectral and spatial resolution(Huang,2014; Hasanlou,2016), extraction of buildings shadow by PSI (shadow index from principal component transformation and RGB transformation ), purification of shadow by wiping off vegetation and water bodies acreage through multi-scale segmentation objects statistic, modelling of shadow and floor area ratio based by 35 real residential mini districts data.

The shadow acreage of the buildings is positively related to the volume of the building, and which can reflect the building floor area ratio, high resolution satellites can detail the height and the width of buildings as well as depth or more other information,. Therefore, the first processing is to accurately extract the shadow of buildings. We get shadow area by the improved shadow index model (Liu,2014), while some vegetation and water mix in the shadows, then, next step is to remove them. Set the threshold of SI minus NDVI to remove vegetation, now, only water area needs to deal with, the method is, we do different scale segmentation to remove different size of water, firstly, with a large scale segmentation to remove large pieces of water body, then, combine and remove small scale of segmentation water pieces. Eventually, we get the purified building shadow acreage with accuracy of $95 \%$.

Inversion building floor ratio by shadow acreage has been a mature technology in recent years, for the characteristics of fast and high efficiency in the estimation of the building volume ratio of large cities, which is one of the main methods of inversion of building volume ratio. The buildings acreage is an important parameter of the relationship between the floor area ratio and the shadow area, In order to obtain the relationship between the shadow area and the total acreage of the buildings, we get total buildings area 34 plot of residential districts by the field surveying and shadow total acreage by remote sensing to do regression analysis, the scatter plots results is shown in Figure 1

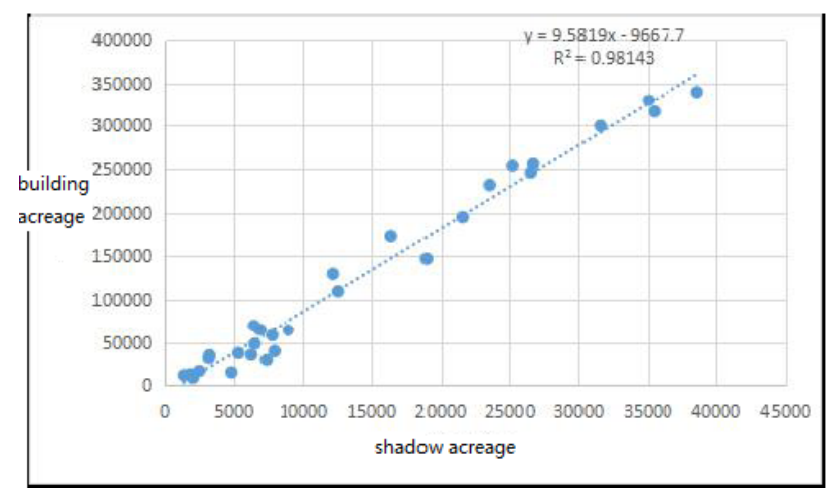

Figure 1 regression analysis of the total building acreage and shadow acreage.

You can see a good linear relationship between the total area of the buildings and the shadow area. The deterministic coefficient is very close to 1 , this means that, we can calculate the very reliable total buildings acreage by shadow acreage. The relationship from little residential villages can be used in each street level, with a base of none mountains and lakes acreage interference, and the use of the shadow area of each street can 
infer out the total area of the buildings, and then floor volume rate of each unit can be obtained through being divided by the land acreage. Among them, because some streets may contain large tracts of mountains, or big water body, so the entire street area should be subtracted by the acreage of them, after we statistics their area, and then the volume rate of the streets level ends calculation. In Arc-GIS, add attribution data to Nanjing vector map, and make the distribution map of each street building volume rate, the overview distribution of the floor volume rate is shown in Figure 2, we use layered colorization method to show them. From the figure 2, we can see that, the old town, commercial centre area, such as streets in Gulou District, Jianye District, Qinhuai District has the largest building volume rate, they are more than 3, that of new exploited units and old urban edge is relative low. On the other hand, industry land has low building volume rate, for example, Molin Street of Jiangning Development Zone has moderate building floor rate, for it is planned for industry and residential usage. From the figure and the model parameter, we can obtain precisely building volume rate from ZY-3 images, thus help to ensure the effect of the next step works.

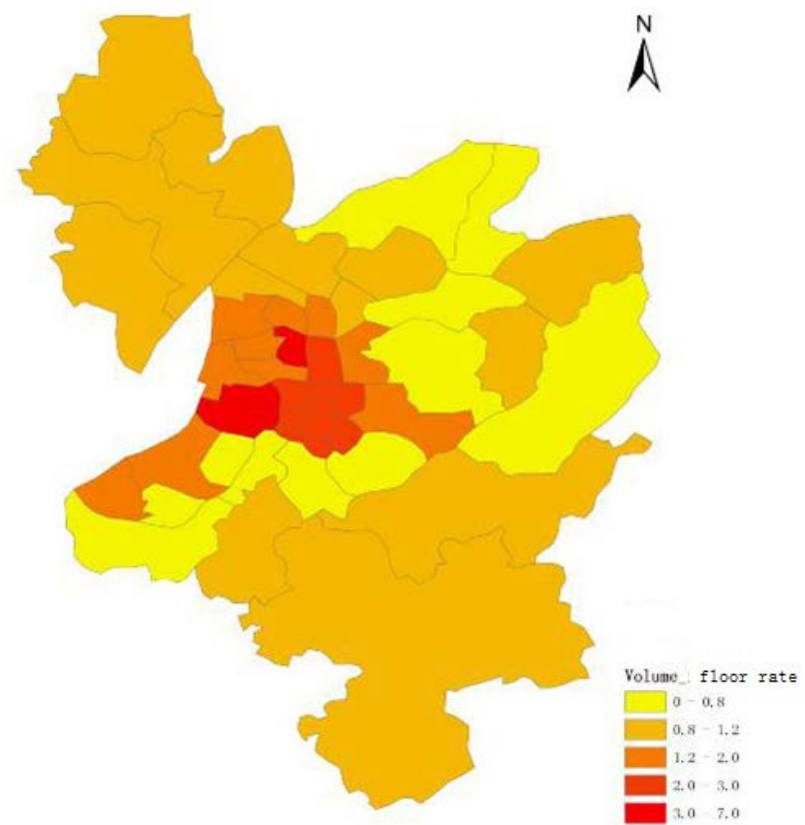

Figure 2 map of building volume rate for each street

\subsection{Relationship Modelling}

We do two jobs to complete this goal, one is getting the nightlight index by averaging data of 12 months and then averaging them in a whole administrative level street, the other is modelling the regression relationship between the floor area ratio and the nightlight index.

The nightlight data for the 12 months of 2015 of all streets in Nanjing is averaged, the scope and statistics the streets of the VIIRS data of each street is extracted by their shape maps restriction. The mean brightness of each pixel of each street is calculated as nightlight index. The light index and the volume rate are used to regression analysis, the best deterministic coefficient model and the scatter diagram is shown in Figure3.

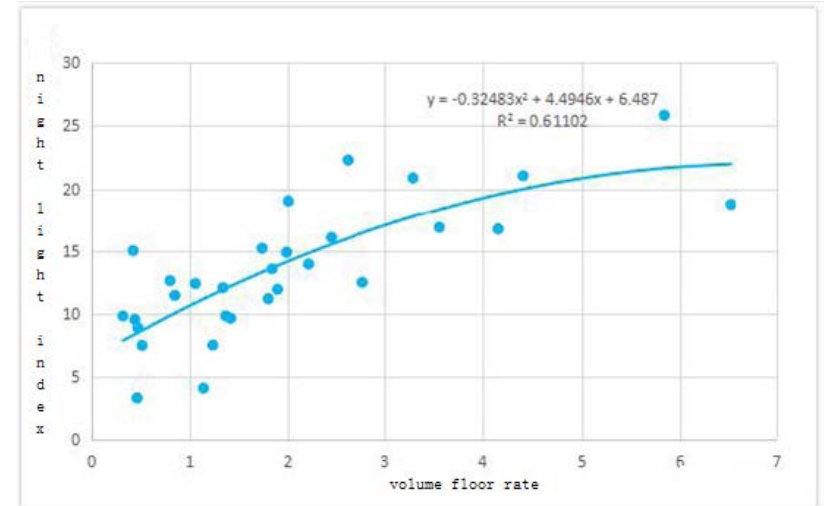

Figure 3. Regression analysis of the building volume floor rate and night light index.

It can be found that the relationship between the light index and the volume ratio is obvious, and the correlation of the polynomial regression is highest than that of other regression models. With the increasing of the volume ratio, the light index increases small gradually and gradually before it reaches the pole value. That means, nightlight index will saturate and smooth down when the building volume rate is greater than or equal to 6. The distribution of the light index (shown in Figure 2 on the right) and the volume ratio for comparing is shown in figure3.

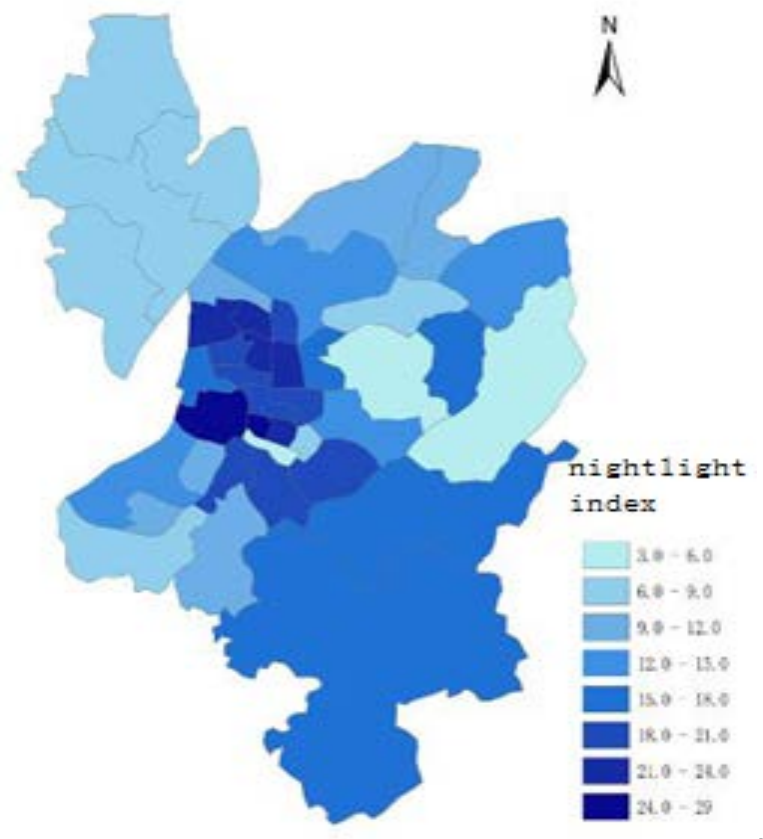

Figure 3 map of nightlight index for each street

\section{RESULTS AND ANALYSIS}

As for the relationship between the shadow and the floor area ratio, testing data of 20 residential mini districts shows that there is a lineal relationship and the $\mathrm{R}^{2}$ is 0.98 . As for the relationship between the floor area ratio and the nightlight, it shows that there is a quadratic polynomial relationship and the $\mathrm{R}^{2}$ is 0.611 . The two regression functions of this article are showed in following Table 1. 


\begin{tabular}{|l|l|l|c|}
\hline & \multicolumn{1}{|c|}{ Expression function } & \multicolumn{1}{|c|}{$\begin{array}{c}\text { Dependent } \\
\text { variable }\end{array}$} & $\mathrm{R}^{2}$ \\
\hline Formula 1 & $\begin{array}{l}\mathrm{Y}=9.5819 \mathrm{x}-9667.7 \\
\mathrm{Y}=-.32483 \mathrm{x}^{2}+4.4946 \mathrm{x} \\
+6.487\end{array}$ & $\begin{array}{l}\text { Shadow } \\
\text { Nightlight } \\
\text { index }\end{array}$ & 0.98 \\
\hline
\end{tabular}

Table 1. The two regression functions

We can see that, floor area ratio obtained from the shadow is quite accurate, for the geometry information is clear in high resolution images, Floor area ratio and shadow have lineal expression, for shadow area is proportional to the area of buildings; while the floor area ratio obtained from the nightlight index is more uncertainty, for nightlight information is easily disturbed and damped by other factors, such as clouds, frost, and other chance events etc., especially, lights of upper floor will conceal lights of down floors; moreover, it will saturate when the lights adding to an extent, which conduct to an exceeding of the sensitivity of the remote sensor, all these cause the results that nightlight index and floor area ratio have quadratic polynomial expression.

From the figures of volume rate distribution and the nightlight distribution, we can find out that, in the prosperous center of the city, such as streets of Gulou Districts and Jianye Districts, both volume rate and light index are high, and those of the surrounding areas are lower, as for Jiangning District, who is in better development style, its volume rate and light index are all in a moderate position. The nightlight index is not fully correspond to the buildings volume rate in high-rise forest area, the reason is the overlay and overlay by each other.

The level of building floor volume rate and the nightlight index is relatively synchronize, the heavier the night light value of the area, the higher the building floor area ratio, it is square polynomial function, that is, the growth rate is slower than the volume rate, and gradually tend to an extreme point. The increase in floor area ratio does not lead to an infinite increase in the light index which is mainly because of high-rise lighting superimposed effect, with additional traffic networks streetlights and other factors impaction on the city. According to this, we can draw the rule: average night light brightness of the street is limited to a certain extent.

\section{CONCLUSION}

From the results and the processing of buildings volume rate from ZY-3 images and from VIIRS nightlight data, they are all usable in different certain content. We can get a conclusion though the model and the deterministic coefficient that, ZY-3 images can imply the floor area ratio inversion of urban street administrative level after precisely shadow extraction; VIIRS/NPP nightlights data may show the floor area ratio in an extent after some proceeding. In future, we can try to improve the efficiency by two methods, one is mending the nightlight index accumulation method, the other is merging the nightlight index with the high resolution remote sensing images classification results. Nightlight data will contribute more in urban cognition and analysis.

\section{ACKNOWLEDGEMENTS}

Xiaoyu Li, Beibei Wang, Kaizhe Xu,Huiyao Dai, Zhiheng Liu and Zhaoliang Liu help to processing the images and data.
This research was funded by 'the Fundamental Research Funds for the Central Universities'- Considering Population Density of Eco-environment Quality Evaluation of Urban Habitat Based on High Resolution.

\section{REFERENCES}

Dc, D. O. C. W., \& Census Bureau, W. D. (2003). United states census 2000: census of population and housing. summary file 2.

Forbes, D. J. 2011. Statistical correlation between economic activity and DMSP/OLS night light images in florida. Dissertations \& Theses - Gradworks.

Hasanlou, M., \& Saradjian, M. R. 2016. Quality assessment of pan-sharpening methods in high-resolution satellite images using radiometric and geometric index. Arabian Journal of Geosciences, 9(1), 1-10.

Hillger D,T Kopp,T Lee,et al. 2013,First-Light imagery from Suomi NPP VIIRS[J]. Bulletin of the American Meteorological Society, 94(7):1019-1029.

Huang X, Wen D, Xie J, et al. 2014,Quality Assessment of Panchromatic and Multispectral Image Fusion for the ZY-3 Satellite: From an Information Extraction Perspective[J]. Geoscience \& Remote Sensing Letters IEEE, 11(4):753-75.

Kellner, J. R., \& Hubbell, S. P. 2017. Adult mortality in a lowdensity tree population using high-resolution remote sensing. Ecology.

Liu H. 2014,Extraction of the Floor Area Ratio in the Central District of Fuzhou cityBased on Improved Shadow Index Model.Geormatics and inforation science of Wuhan University, 39(10):1241-1218.

Qin, X., Wei, Y., Chen, W., \& Duan, X. 2013. Population expansion and polycentric development of nanjing city in a period of hyper-growth. Geographical Research, 32(4), 711719.

Small C C D 2013, Elvidge.Night onearth: Mapping decadal changes of anthropogenic night light in Asia[J]. International Journal of Applied Earth Observation and Geoinformation, 22(6):40-52.

Townsend A C,D A 2010 , Bruce.The use of night-time lights satellite imagery as a measure of Australia's regional electricity consumption and population distribution[J]. International Journal of Remote Sensing, 31(16 ) : 44594480 .

Vermote E, Justice C, Csiszar I. 2014,Early evaluation of the VIIRS calibration, cloud mask and surface reflectance Earth data records[J]. Remote Sensing of Environment, 148(6):134-145. 